\title{
Arterial Lactate Concentration At The End of Liver Transplantation is Independently Associated with One-Year Mortality
}

\section{Sean Coeckelenbergh}

Université Libre de Bruxelles

Leonard Drouard

Université Libre de Bruxelles

\section{Brigitte Ickx}

Université Libre de Bruxelles

\section{Valerio Lucidi}

Cliniques Universitaires de Bruxelles, Université Libre de Bruxelles

\section{Dessy Germanova}

Cliniques Universitaires de Bruxelles, Université Libre de Bruxelles

\section{Olivier Desebbe}

Sauvegarde Clinic

\section{Maya Moussa}

Hôpitaux Universitaires Paris-Sud, Université Paris-Sud, Université Paris-Saclay, Paul Brousse Hospital, Assistance Publique Hôpitaux de Paris (APHP)

\section{Salima Naili}

Hôpitaux Universitaires Paris-Sud, Université Paris-Sud, Université Paris-Saclay, Paul Brousse Hospital, Assistance Publique Hôpitaux de Paris (APHP)

\section{Eric Vibert}

Hôpitaux Universitaires Paris-Sud, Université Paris- Sud, Université Paris-Saclay, Paul Brousse Hospital, Assistance Publique Hôpitaux de Paris (APHP)

\section{Didier Samuel}

Hôpitaux Universitaires Paris-Sud, Université Paris-Sud, Université Paris-Saclay, Paul Brousse Hospital, Assistance Publique Hôpitaux de Paris (APHP)

\section{Jacques Duranteau}

Hôpitaux Universitaires Paris-Sud, Université Paris-Sud, Université Paris-Saclay, Paul Brousse Hospital, Assistance Publique Hôpitaux de Paris (APHP)

\section{Jean-Louis Vincent}

Université Libre de Bruxelles Joseph Rinehart

University of California Irvine 
Philippe Van der Linden

Université Libre de Bruxelles

Alexandre Joosten ( $\nabla$ joosten-alexandre@hotmail.com )

Université Libre de Bruxelles

\section{Research Article}

Keywords: Hemodynamics, goal-directed hemodynamic therapy, postoperative outcome, high-risk abdominal surgery, liver surgery.

Posted Date: June 23rd, 2021

DOI: https://doi.org/10.21203/rs.3.rs-601997/v1

License: (c) (1) This work is licensed under a Creative Commons Attribution 4.0 International License.

Read Full License 


\section{Abstract}

\section{BACKGROUND}

Liver transplant patients who develop hyperlactatemia are at increased risk of postoperative morbidity and mortality, but there are few data on longer-term outcomes. We therefore investigated whether arterial lactate concentration obtained immediately after surgery, at the time of admission to the intensive care unit (ICU), was associated with 1-year mortality.

\section{METHODS.}

In this retrospective cohort study, all patients who underwent liver transplant surgery between September 2013 and December 2019 were screened for inclusion. Patients who underwent combined transplantation surgery and those with a history of previous liver transplantation (i.e., redo surgery) were not included. Logistic regression modeling included univariate and multivariate analyses. Receiver operating characteristic (ROC) curves and areas under the curves (AUROCs) were calculated. Lactate thresholds and association with outcome were analyzed for specificity, sensitivity, and Youden's index.

RESULTS. Of 226 patients included, $18.4 \%$ died within 1-year of liver transplantation. Immediate postoperative lactate concentration was independently associated with 1-year mortality with an odds ratio (OR) of 1.35 (95\% Cl: 1.16 to $1.59 ; \mathrm{p}<0.001)$ per $\mathrm{mEq} / \mathrm{L}$ increase in lactate and an AUROC of 0.80 ( $95 \% \mathrm{Cl}: 0.72$ to $0.87 ; \mathrm{p}<0.001$ ). A lactate concentration of $2.25 \mathrm{mEq} / \mathrm{L}$ (cut-off determined using Youden's index) was associated with increased 1-year mortality with a sensitivity of 0.71 and a specificity of 0.72 .

CONCLUSION: Increased arterial lactate concentration on admission to the ICU immediately after orthotopic liver transplantation is independently associated with increased 1-year mortality.

\section{Trial Registration: Not Applicable}

\section{Background}

Blood lactate concentrations reflect an imbalance between systemic oxygen delivery and tissue oxygen demand, which results in anaerobic metabolism.[1] Because about $60 \%$ of lactate is cleared by the liver, blood lactate concentrations also provide an indication of hepatic blood flow and function.[2] Raised blood lactate concentrations may therefore reflect intra-operative tissue dysoxia and graft dysfunction in liver transplant patients.[3-6] Rarer causes of hyperlactatemia include enzymatic (e.g., pyruvate dehydrogenase) alterations, pharmacologic effects (e.g., metformin), and hypothermia.[1] Hyperlactatemia is often associated with metabolic acidosis, which can alter a patient's hemostasis and hemodynamics, worsen hemorrhage, exacerbate postoperative complications, and ultimately increase mortality. $[1,7]$ Normal arterial lactate concentrations are around $1 \mathrm{mEq} / \mathrm{L}$ and values greater than 1.5 $\mathrm{mEq} / \mathrm{L}$ have been associated with increased mortality in various studies of surgical and critically ill patients.[8-10] Blood lactate levels may therefore represent a useful predictor of complications and outcomes in liver transplant patients.[10] 
Although the presence of hyperlactatemia at the end of liver transplantation has been shown to be associated with postoperative mortality within the first [11] and third [4] months after liver transplantation, there are few data on its relationship with long-term mortality. The primary objective of this cohort study was therefore to test the hypothesis that increased lactate concentration on after liver transplantation is associated with increased 1-year mortality.

\section{Methods}

The Erasme Hospital Institutional Review Board approved this monocenter study on December 14, 2018 (reference P2018/555), and waived the need for patient informed consent because of the study's retrospective nature. Using our anesthetic electronic records software (Innovian, Drager, Germany), we identified all patients who had had an orthotopic liver transplant between September 2013, date of implementation of the software, and December 2019. We reviewed the electronic medical records (MediView, IMMJ Systems, United Kingdom) of all these patients and included those for whom operating room planning dedicated software (TrackPro, UltraGenda, Belgium) had been used. Exclusion criteria included incomplete records, combined surgeries (e.g., simultaneous lung and liver transplant), and previous liver transplantation.

\section{Anesthesia protocol}

Anesthesia was standardized according to our institutional protocol. After arrival in the operating room, patients and were placed under an infrared heating lamp. Standard non-invasive monitoring consisted of a 5-lead EKG, non-invasive blood pressure, temperature, frontal processed electroencephalogram, diuresis, and neuromuscular blockade monitoring. Vascular access consisted of one or two large bore peripheral venous catheters, right femoral artery and vein catheters, and right jugular vein catheter. The left femoral and internal jugular veins were left at the surgeon's disposal for possible veno-venous bypass. A pulmonary artery (Swan-Ganz) catheter was inserted, and hemodynamic management was guided using calibrated cardiac index, central venous oxygen saturation, inferior vena cava pressure, central venous pressure, and arterial pressure. Hemodynamic targets were left to the discretion of the attending anesthesiologist. Rapid infusers, perfusion heaters, and an intraoperative blood product salvage system were ready for use prior to induction. In case of massive bleeding, it was recommended that the anesthesiologist should use ROTEM technology to guide blood product administration. Anesthesia was induced with propofol or etomidate and neuromuscular blockade with rocuronium or succinylcholine, depending on the patient's history, and maintained with cisatracurium. Anti-nociception was maintained with remifentanil and unconsciousness with sevoflurane or desflurane. Rapid sequence intubation was performed if patients had not fasted or had abdominal ascites.

\section{Surgical procedure}

The standard approach for orthotopic liver transplantation was recipient hepatectomy with a vena cavasparing technique, piggy-back reconstruction, and no venous-venous bypass. Liver reperfusion began with the portal vein and then arterial reperfusion. End-to-end choledochectomy without a T-tube assured 
biliary reconstruction. Portal ischemia (i.e., cold ischemia) time was defined as the time from donor aortic clamping to portal unclamping of the recipient. Arterial ischemia (i.e., warm ischemia) was defined as the time from recipient portal unclamping to hepatic artery unclamping. Immunosuppression consisted of tacrolimus with mycophenolate mofetil and prednisone. Tacrolimus levels were targeted at $5-10 \mathrm{ng} / \mathrm{mL}$ and steroids were maintained for the first 3 months post liver transplantation.

At our institution all patients are transferred to the ICU immediately after liver transplantation.

\section{Data collection and outcomes}

Preoperative demographics, intraoperative and postoperative (at patient arrival in the ICU) arterial lactate concentrations, postoperative major and minor complications, 30-day mortality, and 1-year mortality were collected from the medical records for each patient.

The primary objective was to test the hypothesis that postoperative lactate concentration at ICU admission was associated with long-term mortality (i.e., death within one year of transplantation). Secondary objectives included the association between lactate concentration and 30-day mortality and between lactate and major postoperative complications (defined in Appendix 1).

\section{Statistical Analysis}

Data are reported as mean \pm standard deviation or median [25th -75th percentiles] depending on the normality of the distribution as tested by the Shapiro-Wilk test. Results for counts are reported as number (\% of group). Baseline, intraoperative, and outcome data were first compared groupwise with a Student's t-test or a Mann-Whitney $U$ for normal and skewed data, respectively, or a chi-square test for counts. Univariate testing identified potential contributors to mortality. All variables with a $p$ value $<0.10$ were introduced into a multivariate analysis and a backwards feature selection process removed variables with a $p$ value $>0.05$ in the model.

Receiver operating characteristic (ROC) curves were established for 1-year mortality, 30-day mortality, and postoperative complications; 95\% confidence intervals were calculated using the DeLong method. Lactate concentration thresholds and the mortality or morbidity risk were calculated, with 2000 bootstrapped Monte-Carlo samples for specificity, sensitivity, and Youden's Index

\section{Results}

A total of 276 liver transplant procedures were performed during the study period; 28 patients had incomplete anesthesia medical records, 10 had had combined surgery, and 10 had had a previous liver transplant. Accordingly, 228 patients were included in the analysis (Fig. 1). Forty-two patients (18.6\%) patients died within the year after transplantation

The American Society of Anesthesiology (ASA) score, Child-Pugh score, Model for End-stage Liver Disease (MELD) score, duration of anesthesia, duration of surgery, crystalloid volume, blood product transfusion, platelet transfusion, total infused volume, fluid balance, portal ischemia time, mean end tidal $\mathrm{CO}_{2}$, and immediate postoperative lactate concentration were associated with 1-year mortality in 
univariate analysis (Tables 1 and 2). Postoperative events associated with 1-year mortality were: sepsis, postoperative bleeding requiring transfusions, atrial fibrillation, encephalopathy, acute respiratory distress syndrome, reoperation, renal replacement therapy, and acute kidney injury (stages II and III) (Table 3). 
Table 1

Baseline Characteristics

\begin{tabular}{|c|c|c|c|}
\hline & Deceased at 1-year & Alive at 1-year & P-value \\
\hline & $(N=42)$ & $(N=184)$ & \\
\hline Male (\%) & $29(69.0 \%)$ & $131(71.2 \%)$ & 0.93 \\
\hline Age (years) & $56.9 \pm 9.64$ & $54.9 \pm 10.4$ & 0.24 \\
\hline Donor Age (years) & $56.7 \pm 16.6$ & $53.9 \pm 15.8$ & 0.33 \\
\hline Weight (Kg) & $82.7 \pm 22.7$ & $79.7 \pm 19.1$ & 0.43 \\
\hline Height $(\mathrm{cm})$ & $172 \pm 9.59$ & $171 \pm 11.3$ & 0.71 \\
\hline $\operatorname{BSA}\left(m^{2}\right)$ & $1.95 \pm 0.27$ & $1.91 \pm 0.25$ & 0.37 \\
\hline ASA score & & & 0.0048 \\
\hline 1 & $0(0 \%)$ & $0(0 \%)$ & \\
\hline 2 & $1(2.4 \%)$ & $5(2.7 \%)$ & \\
\hline 3 & $12(28.6 \%)$ & $105(57.1 \%)$ & \\
\hline 4 & $27(64.3 \%)$ & $72(39.1 \%)$ & \\
\hline 5 & $2(4.8 \%)$ & $2(1.1 \%)$ & \\
\hline Child-Pugh Score & $10.6 \pm 3.44$ & $9.11 \pm 3.20$ & 0.017 \\
\hline MELD Score & $24.4 \pm 12.5$ & $19.4 \pm 10.7$ & 0.031 \\
\hline Etiology of liver failure & & & 0.14 \\
\hline Acute hepatic failure & $7(16.7 \%)$ & $8(4.3 \%)$ & \\
\hline Biliary disease & $4(9.5 \%)$ & $12(6.5 \%)$ & \\
\hline Thrombotic & $0(0 \%)$ & $3(1.6 \%)$ & \\
\hline Cirrhosis & $13(31.0 \%)$ & $73(39.7 \%)$ & \\
\hline Malignancy & $12(28.6 \%)$ & $70(38.0 \%)$ & \\
\hline $\mathrm{NASH}$ & $5(11.9 \%)$ & $5(2.7 \%)$ & \\
\hline Benign tumor & $0(0 \%)$ & $3(1.6 \%)$ & \\
\hline Hepato-pulmonary syndrome & $0(0 \%)$ & $2(1.1 \%)$ & \\
\hline
\end{tabular}

Data are presented as mean \pm SD or number (\%). statistically significant values are in bold ASA American Society of Anesthesiology BSA Body Surface Area MELD Model for End-stage Liver Disease 


\begin{tabular}{|llll}
\hline & Deceased at 1-year & Alive at 1-year & P-value \\
\cline { 2 - 3 } & $\mathbf{( N = 4 2 )}$ & $\mathbf{( N = 1 8 4 )}$ & $1(0.5 \%)$ \\
\hline Polycystic disease & $0(0 \%)$ & $1(0.5 \%)$ \\
\hline Other & $0(0 \%)$ & $7(3.8 \%)$ \\
\hline Unknown & $1(2.4 \%)$ &
\end{tabular}

Data are presented as mean \pm SD or number (\%). statistically significant values are in bold

ASA American Society of Anesthesiology BSA Body Surface Area MELD Model for End-stage Liver Disease 
Table 2

Intraoperative events

\begin{tabular}{|llll|}
\hline & Deceased at 1-year & Alive at 1-year & P-value \\
\cline { 2 - 3 } & $\mathbf{( N = 4 2 )}$ & $\mathbf{( N = 1 8 4 )}$ & \\
\hline Key durations & & & \\
\hline Duration of anesthesia & $566 \pm 202$ & $500 \pm 106$ & 0.046 \\
\hline Duration of surgery & $402 \pm 132$ & $367 \pm 85.6$ & 0.10 \\
\hline Duration of portal ischemia & $453 \pm 116$ & $411 \pm 104$ & 0.037 \\
\hline Duration of arterial ischemia & $40.1 \pm 24.0$ & $35.2 \pm 20.1$ & 0.23 \\
\hline Infused fluids & & & \\
\hline Crystalloid & $3540 \pm 3000$ & $2680 \pm 1720$ & 0.078 \\
\hline Colloid & $1060 \pm 889$ & $867 \pm 885$ & 0.2 \\
\hline Bicarbonate & $81.2 \pm 237$ & $31.2 \pm 70.8$ & 0.18 \\
\hline Pack Red Blood Cells & $2790 \pm 2680$ & $1830 \pm 2230$ & 0.036 \\
\hline Plasma & $1190 \pm 1170$ & $875 \pm 1150$ & 0.12 \\
\hline Platelets & $350 \pm 397$ & $184 \pm 308$ & 0.014 \\
\hline Cell Saver Return & $623 \pm 1050$ & $646 \pm 1040$ & 0.89 \\
\hline Total in & $10900 \pm 8210$ & $7900 \pm 5610$ & $\mathbf{0 . 0 2 9}$ \\
\hline Fluid loss & & $30.7 \pm 3.61$ & 0.063 \\
\hline Estimated Blood Loss & $4350 \pm 4670$ & $3140 \pm 3100$ & 0.11 \\
\hline Urine Output & $386 \pm 363$ & $378 \pm 362$ & 0.89 \\
\hline Total Out & $4740 \pm 4670$ & $3520 \pm 3130$ & 0.11 \\
\hline Net fluid balance & $6150 \pm 5760$ & $4380 \pm 4260$ & 0.066 \\
\hline Organ Perfusion Parameters & & & \\
\hline Average MAP & $72.2 \pm 7.22$ & $73.3 \pm 7.09$ & 0.38 \\
\hline Time with MAP $<65 m m H g$ & $33.3 \pm 20.3$ & $28.1 \pm 20.4$ & 0.14 \\
\hline End-Tidal CO 2 & & & \\
\hline & & & \\
\hline
\end{tabular}

Data are presented as mean \pm SD. Statistically significant values are in bold ICU Intensive Care Unit MAP mean arterial pressure 


\begin{tabular}{|llll|}
\hline & Deceased at 1-year & Alive at 1-year & P-value \\
\cline { 2 - 4 } & $(\mathbf{N}=\mathbf{4 2})$ & $\mathbf{( N = 1 8 4 )}$ & \\
\hline Lactate on ICU admission & $6.6 \pm 5.9$ & $2.3 \pm 2.11$ & $\mathbf{0 . 0 0 0 0 4}$ \\
\hline \multicolumn{4}{|l}{ Data are presented as mean \pm SD. Statistically significant values are in bold } \\
\hline \multicolumn{4}{|l}{ ICU Intensive Care Unit MAP mean arterial pressure } \\
\hline
\end{tabular}


Table 3

Complications and outcomes

Deceased at 1-year

$(\mathrm{N}=42)$
Alive at 1-year

$(\mathrm{N}=184)$

\section{Major complications}

Biliary dysfunction

Anastomotic leakage

Peritonitis

Sepsis

Primary graft failure

Bleeding requiring reoperation

Atrial fibrillation

Acute coronary syndrome

Stroke

Graft thrombosis

DVP/PE

Encephalopathy

ARDS

Heart failure

Reoperation (Any Cause)

Renal replacement therapy

AKI KDIGO II

AKI KDIGO III

Any major complication

\section{Minor Complications}

AKI KDIGO I
$12(28.6 \%)$

$8(19.0 \%)$

$3(7.1 \%)$

$13(31.0 \%)$

$3(7.1 \%)$

25 (59.5\%)

$9(21.4 \%)$

$1(2.4 \%)$

0 (0\%)

$6(14.3 \%)$

$2(4.8 \%)$

16 (38.1\%)

$12(28.6 \%)$

4 (9.5\%)

$21(50.0 \%)$

23 (54.8\%)

$2(4.8 \%)$

25 (59.5\%)

$5.0[0.0-10.0]$

$7(16.7 \%)$
$40(21.7 \%)$

$11(6.0 \%)$

$4(2.2 \%)$

13 (7.1\%)

7 (3.8\%)

0.59

$43(23.4 \%)$

16 (8.7\%)

1 (0.5\%)

1 (0.5\%)

13 (7.1\%)

$5(2.7 \%)$

16 (8.7\%)

15 (8.2\%)

13 (7.1\%)

36 (19.6\%)

19 (10.3\%)

29 (15.8\%)

29 (15.8\%)

$1.0[0.0-9.0]$

$<0.001$

$>0.99$

0.22

0.84

$<0.001$

$<0.001$

0.82

$<0.001$

$<0.001$

0.10

$<0.001$

Data are presented as IQR [Q25, Q75] or number (\%)

*statistically significant values are in bold

AKI Acute Kidney injury ARDS Acute Respiratory Distress Syndrome KDIGO Kidney Disease: Improving Global Outcomes ; DVP: deep venous thrombosis; PE: pulmonary embolism 


\begin{tabular}{|llll|}
\hline & Deceased at 1-year & Alive at 1-year & P-value \\
\cline { 2 - 4 } & $\mathbf{( N = 4 2 )}$ & $\mathbf{N}=184)$ & \\
\hline Urinary Infection & $1(2.4 \%)$ & $8(4.3 \%)$ & 0.88 \\
\hline Ileus & $9(21.4 \%)$ & $23(12.5 \%)$ & 0.21 \\
\hline Pleural Effusion & $7(16.7 \%)$ & $24(13.0 \%)$ & 0.71 \\
\hline Pneumonia & $4(9.5 \%)$ & $8(4.3 \%)$ & 0.33 \\
\hline Delirium & $13(31.0 \%)$ & $25(13.6 \%)$ & 0.013 \\
\hline Other infection & $10(23.8 \%)$ & $17(9.2 \%)$ & 0.0181 \\
\hline Any Minor Complication & $1.0[0.0-3.0]$ & $0[0.0-4.0]$ & 0.0047 \\
\hline Hospitalization & & $3[1-27]$ & 0.0031 \\
\hline ICU Length of stay & $5[1-27]$ & $15[7-166]$ & 0.16 \\
\hline Hospital Length of stay & $23[2-122]$ & $12(6.5 \%)$ & 0.38 \\
\hline Readmission within 90 days & $5(11.9 \%)$ & & $<0.001$ \\
\hline Mortality & & & \\
\hline 1-Year mortality & $42(100 \%)$ & & \\
\hline Data are presented as IQR [Q25, Q75] or number (\%) & & \\
\hline *statistically significant values are in bold & & \\
\hline AKI Acute Kidney injury ARDS Acute Respiratory Distress Syndrome & KDIGO Kidney Disease: Improving \\
\hline Global Outcomes; DVP: deep venous thrombosis; PE: pulmonary embolism & \\
\hline
\end{tabular}

In the multivariate model, ASA score, duration of anesthesia, infused crystalloid volume, total infused volume, and lactate concentration at ICU admission were independently associated with 1-year mortality (Table 4). Lactate concentration at ICU admission was strongly associated with 1-year mortality with an odds ratio (OR) of 1.35 (95\% Cl: 1.16 to $1.59 ; \mathrm{p}<0.001)$ per unit increase in lactate. The AUROC was 0.80 (95\% Cl: 0.72 to $0.87 ; p<0.001$ ) (Fig. 2), and a lactate concentration of $2.25 \mathrm{mEql} / \mathrm{L}$ (cut-off determined using Youden's index) was associated with 1-year mortality with a sensitivity of 0.71 and a specificity of 0.72. Lactate concentration at ICU admission also predicted 30-day mortality, with an AUROC of 0.91 ( $95 \% \mathrm{Cl}: 0.84$ to $0.97 ; \mathrm{p}<0.0001$ ). A lactate concentration of $2.65 \mathrm{mEq} / \mathrm{L}$ was associated with 30 -day mortality with a sensitivity of 0.94 and a specificity of 0.72 . Lactate concentration predicted major postoperative complications poorly, with an AUROC of 0.63 (95\% Cl: 0.56 to $0.71 ; p=0.0013$ ). A lactate concentration of $2.55 \mathrm{mEq} / \mathrm{L}$ was associated with major postoperative complications with a sensitivity of 0.41 and a specificity of 0.80 . 
Table 4

Factors independently associated with postoperative mortality

\begin{tabular}{|llll|}
\hline Factor & $\begin{array}{l}\text { Adjusted } \\
\text { Odds Ratio }\end{array}$ & $95 \% \mathrm{Cl}$ & P value \\
\hline ASA Score & 2.4040 & $1.0703-5.3994$ & $\mathbf{0 . 0 3 3}$ \\
\hline Anesthesia duration (per minute) & 1.0037 & $1.0004-1.0071$ & $\mathbf{0 . 0 3 0}$ \\
\hline Intraoperative crystalloid (per ml) & 1.0006 & $1.0001-1.0010$ & $\mathbf{0 . 0 0 8}$ \\
\hline Intraoperative total infused volume (per ml) & 0.9996 & $0.9992-0.9999$ & $\mathbf{0 . 0 3 5}$ \\
\hline Lactate on ICU Admission (per mEq/L) & 1.3568 & $1.1590-1.5884$ & $<\mathbf{0 . 0 0 1}$ \\
\hline ASA - American Society of Anesthesiology; ICU - Intensive Care Unit & \\
\hline
\end{tabular}

\section{Discussion}

Even a moderate increase in blood lactate concentration early after orthotopic liver transplantation was associated with increased long-term mortality. Previous studies have reported an association between postoperative hyperlactatemia and mortality after liver transplantation,[4, 11, 12] but none evaluated longterm mortality. Our study is consistent with the prior observations after liver transplantation[4] liver surgery,[12] major abdominal surgery,[13] cardiac surgery [14-16], and intensive care admission.[9]

There are several potential causes of increased lactate concentration at the end of liver transplant surgery. Liver transplant patients can develop tissue dysoxia and graft failure, both of which have been associated with increased mortality during major abdominal surgery and after liver transplantation.[5, 6] However, the incidence of graft failure in our cohort was similar in survivors and non-survivors, suggesting that tissue hypoperfusion was the predominant cause of the higher lactate concentrations in the non-survivors. Other factors contributing to hyperlactatemia could be compromised hemodynamic status during complex surgery, reflected by increased surgical duration and portal ischemia. Hemodynamic alterations can lead to tissue hypoxia with subsequent development of anaerobic metabolism [17] and followed by multiple organ dysfunction.[18] The significantly higher rates of organ failure, including encephalopathy, acute respiratory distress syndrome, kidney injury, and sepsis in patients who did not survive the first year after transplantation compared to those who did, further suggests that intraoperative tissue dysoxia contributed to the increased lactate concentrations. Patients who died within one year of transplantation had higher preoperative risk (i.e., higher ASA, MELD, and Child-Pugh scores). These scores reflect patient frailty and are linked to hepatic and other organ dysfunctions, which increase the burden of perioperative patient care. [19-21] The ASA score, for example, has been consistently linked with increased postoperative complications and mortality.[21] Our results confirm this finding, as the ASA score was independently associated with postoperative mortality. 
Several other factors were independently associated with postoperative mortality, including duration of anesthesia and infused crystalloid volume. Excessive fluid administration has been linked to increased postoperative complications either because this is an indicator of hemodynamic instability or because of an intrinsic effect of fluid.[22-24] These factors attest to the hemodynamic and anesthetic challenges of these patients and strengthen the association between unstable hemodynamics, decreased perfusion, tissue hypoxia, increased lactate, and postoperative mortality. Increased postoperative lactate concentration was also associated with major complications, with good specificity. In addition to tissue hypoxia, other causes of postoperative morbidity after liver transplant, include coagulopathy and biliary complications,) $[25,26]$ for which increased lactate concentrations may have lower sensitivity.

Liver transplant patients with postoperative hyperlactatemia should be carefully investigated to determine the cause of hyperlactatemia and administer effective treatment. A perioperative goal-directed hemodynamic strategy may help to prevent intraoperative tissue hypoxia and its associated postoperative complications.[27-29] Maintaining optimal hemodynamic goals (e.g.preload, arterial pressure, and cardiac output), is essential, but clinicians must still recognize that hyperlactatemia may be due to other causes during liver surgery. [12] Regardless of its etiology, hyperlactatemia is linked to increased short- and long-term mortality and its presence should promote close observation and prophylactic measures to prevent postoperative complications and death.

\section{Limitations}

This study had several limitations. First, it was a single center, retrospective study, with a relatively small number of patients. Second, although a single arterial lactate concentration was strongly associated with 30-day and 1-year mortality, evaluating the time course of blood lactate levels may give even more information; this should be further studied. Third, our study did not include donor data, which could have an impact on survival. Although invasive hemodynamic monitors were used, future studies should assess the impact of goal-directed hemodynamic strategies on postoperative morbidity and mortality in liver transplant patients. Our study, however, reflects standard of care in an academic center with expertise in liver transplantation anesthesia.

\section{Conclusions}

In patients who underwent orthotopic liver transplantation, an increased lactate concentration immediately after surgery was independently associated with increased 1-year mortality.

\section{List Of Abbreviations}

ASA

American Society of Anesthesiology

AUC

Area Under the Curve 
ICU

Intensive Care Unit

MELD

Model for End-stage Liver Disease

ROC

Receiver Operating Characteristic

\section{Declarations}

Ethics approval: Study approved by the Institutional Review Board of Erasme hospital on December 14, 2018 under the reference P2018/555.

Consent for publication: Not Applicable

Availability of data and materials: By request to the corresponding author

Competing interests:

$\mathrm{SC}$ is a consultant for Medtronic (Dublin, Ireland)

AJ is a consultant for Edwards Lifesciences (Irvine, California, USA).

OD is a consultant for Medtronic (Dublin, Ireland)

The other authors have no conflicts of interest related to this article

Funding: The authors received no funding for this work

Authors' contributions: All authors read and approved the final manuscript

S.C.: Designed the study, collected and analyzed the data and drafted the manuscript

L.D: Collected and analyzed the data and edited the final manuscript

B.I: Analyzed the data and edited the final manuscript

V.L: Analyzed the data and edited the final manuscript

D.G: Analyzed the data and edited the final manuscript

O.D: Analyzed the data and edited the final manuscript

M.M: Analyzed the data and edited the final manuscript

S.N: Analyzed the data and edited the final manuscript 
E.V: Analyzed the data and edited the final manuscript

D.S: Analyzed the data and edited the final manuscript

J.D: Analyzed the data and edited the final manuscript

JL.V: Analyzed the data and edited the final manuscript

J.R: Statistical analysis of the data and edited the final manuscript.

P.VdL: Statistical analysis of the data and edited the final manuscript.

A.J: Designed the study, collected and analyzed the data and drafted the manuscript.

Acknowledgements: All the clinicians who helped in data collection for the current liver transplant database.

\section{References}

1. Kraut JA, Madias NE: Lactic acidosis. The New England journal of medicine 2014, 371(24):23092319.

2. Vincent JL, Quintairos ESA, Couto L, Jr., Taccone FS: The value of blood lactate kinetics in critically ill patients: a systematic review. Critical care (London, England) 2016, 20(1):257.

3. De Gasperi A, Mazza E, Corti A, Zoppi F, Prosperi M, Fantini G, Scaiola A, Colella G, Amici O, Notaro P et al: Lactate blood levels in the perioperative period of orthotopic liver transplantation. International journal of clinical \& laboratory research 1997, 27(2):123-128.

4. Golse N, Guglielmo N, El Metni A, Frosio F, Cosse C, Naili S, Ichaï P, Ciacio O, Pittau G, Allard MA et al: Arterial Lactate Concentration at the End of Liver Transplantation Is an Early Predictor of Primary Graft Dysfunction. Annals of surgery 2019, 270(1):131-138.

5. Chen XB, Xu MQ: Primary graft dysfunction after liver transplantation. Hepatobiliary \& pancreatic diseases international: HBPD INT 2014, 13(2):125-137.

6. de Jaeger A, Proulx F, Yandza T, Dugas MA, Boeuf B, Manika A, Lacroix J, Lambert M: Markers of cellular dysoxia during orthotopic liver transplantation in pigs. Intensive care medicine 1998, 24(3):268-275.

7. Shenkman B, Budnik I, Einav Y, Hauschner H, Andrejchin M, Martinowitz U: Model of trauma-induced coagulopathy including hemodilution, fibrinolysis, acidosis, and hypothermia: Impact on blood coagulation and platelet function. The journal of trauma and acute care surgery 2017, 82(2):287292.

8. Bolondi G, Mocchegiani F, Montalti R, Nicolini D, Vivarelli M, De Pietri L: Predictive factors of short term outcome after liver transplantation: A review. World journal of gastroenterology 2016, 22(26):5936-5949. 
9. Nichol AD, Egi M, Pettila V, Bellomo R, French C, Hart G, Davies A, Stachowski E, Reade MC, Bailey M et al: Relative hyperlactatemia and hospital mortality in critically ill patients: a retrospective multicentre study. Critical care (London, England) 2010, 14(1):R25.

10. Kim DG, Lee JY, Jung YB, Song SH, Lee JG, Han DH, Joo DJ, Ju MK, Choi GH, Choi JS et al: Clinical significance of lactate clearance for the development of early allograft dysfunction and short-term prognosis in deceased donor liver transplantation. Clinical transplantation 2017, 31(12).

11. Cardoso NM, Silva T, Basile-Filho A, Mente ED, Castro-e-Silva O: A new formula as a predictive score of post-liver transplantation outcome: postoperative MELD-lactate. Transplantation proceedings 2014, 46(5):1407-1412.

12. Connolly C, Stättner S, Niederwieser T, Primavesi F: Systematic review on peri-operative lactate measurements to predict outcomes in patients undergoing liver resection. Journal of hepato-biliarypancreatic sciences 2020, 27(7):359-370.

13. Gruttadauria S, Marino IR, Vitale CH, Mandala L, Scott VL, Doria C: Correlation between peri-operative serum lactate levels and outcome in pancreatic resection for pancreatic cancer, preliminary report. Journal of experimental \& clinical cancer research: CR 2002, 21(4):539-545.

14. Matteucci M, Ferrarese S, Cantore C, Cappabianca G, Massimi G, Mantovani V, Rossi MB, Beghi C: Hyperlactatemia during cardiopulmonary bypass: risk factors and impact on surgical results with a focus on the long-term outcome. Perfusion 2020, 35(8):756-762.

15. Murtuza B, Wall D, Reinhardt Z, Stickley J, Stumper O, Jones TJ, Barron DJ, Brawn WJ: The importance of blood lactate clearance as a predictor of early mortality following the modified Norwood procedure. European journal of cardio-thoracic surgery: official journal of the European Association for Cardio-thoracic Surgery 2011, 40(5):1207-1214.

16. Lopez-Delgado JC, Esteve F, Javierre C, Torrado H, Rodriguez-Castro D, Carrio ML, Farrero E, Skaltsa K, Mañez R, Ventura JL: Evaluation of Serial Arterial Lactate Levels as a Predictor of Hospital and Long-Term Mortality in Patients After Cardiac Surgery. Journal of cardiothoracic and vascular anesthesia 2015, 29(6):1441-1453.

17. Levy B: Lactate and shock state: the metabolic view. Current opinion in critical care 2006, 12(4):315321.

18. Vincent JL, De Backer D: Circulatory shock. The New England journal of medicine 2013, 369(18):1726-1734.

19. Asrani SK, Kim WR: Model for end-stage liver disease: end of the first decade. Clinics in liver disease 2011, 15(4):685-698.

20. Neeff H, Mariaskin D, Spangenberg HC, Hopt UT, Makowiec F: Perioperative mortality after nonhepatic general surgery in patients with liver cirrhosis: an analysis of 138 operations in the 2000s using Child and MELD scores. Journal of gastrointestinal surgery: official journal of the Society for Surgery of the Alimentary Tract 2011, 15(1):1-11.

21. Hackett NJ, De Oliveira GS, Jain UK, Kim JY: ASA class is a reliable independent predictor of medical complications and mortality following surgery. International journal of surgery (London, England) 
2015, 18:184-190.

22. Aga Z, Machina M, McCluskey SA: Greater intravenous fluid volumes are associated with prolonged recovery after colorectal surgery: a retrospective cohort study. British journal of anaesthesia 2016, 116(6):804-810.

23. Joosten A, Coeckelenbergh S, Delaporte A, Ickx B, Closset J, Roumeguere T, Barvais L, Van Obbergh L, Cannesson M, Rinehart $\mathrm{J}$ et al: Implementation of closed-loop-assisted intra-operative goal-directed fluid therapy during major abdominal surgery: A case-control study with propensity matching. European journal of anaesthesiology 2018, 35(9):650-658.

24. Joosten A, Raj Lawrence S, Colesnicenco A, Coeckelenbergh S, Vincent JL, Van der Linden P, Cannesson M, Rinehart J: Personalized Versus Protocolized Fluid Management Using Noninvasive Hemodynamic Monitoring (Clearsight System) in Patients Undergoing Moderate-Risk Abdominal Surgery. Anesthesia and analgesia 2019, 129(1):e8-e12.

25. Mehrabi A, Fonouni H, Müller SA, Schmidt J: Current concepts in transplant surgery: liver transplantation today. Langenbeck's archives of surgery 2008, 393(3):245-260.

26. Kochhar G, Parungao JM, Hanouneh IA, Parsi MA: Biliary complications following liver transplantation. World journal of gastroenterology 2013, 19(19):2841-2846.

27. Reydellet L, Blasco V, Mercier MF, Antonini F, Nafati C, Harti-Souab K, Leone M, Albanese J: Impact of a goal-directed therapy protocol on postoperative fluid balance in patients undergoing liver transplantation: a retrospective study. Annales francaises d'anesthesie et de reanimation 2014, 33(4):e47-54.

28. Pearse RM, Harrison DA, MacDonald N, Gillies MA, Blunt M, Ackland G, Grocott MP, Ahern A, Griggs K, Scott $\mathrm{R}$ et al: Effect of a perioperative, cardiac output-guided hemodynamic therapy algorithm on outcomes following major gastrointestinal surgery: a randomized clinical trial and systematic review. JAMA: the journal of the American Medical Association 2014, 311(21):2181-2190.

29. Futier E, Lefrant JY, Guinot PG, Godet T, Lorne E, Cuvillon P, Bertran S, Leone M, Pastene B, Piriou V et al: Effect of Individualized vs Standard Blood Pressure Management Strategies on Postoperative Organ Dysfunction Among High-Risk Patients Undergoing Major Surgery: A Randomized Clinical Trial. JAMA: the journal of the American Medical Association 2017, 318(14):1346-1357.

\section{Figures}


Figure 1

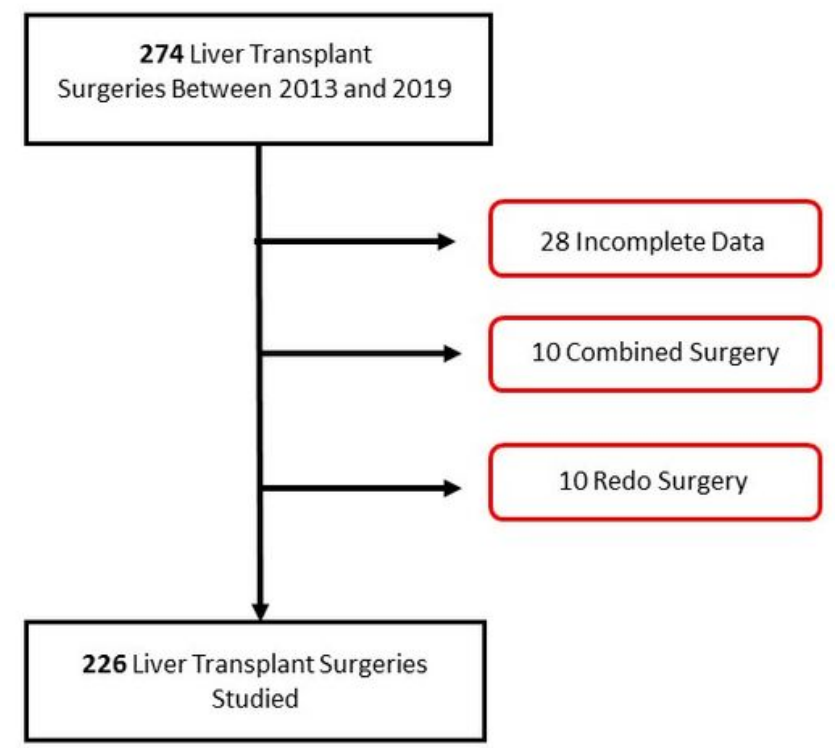

Figure 1

Flow chart of patient inclusion

Figure 2

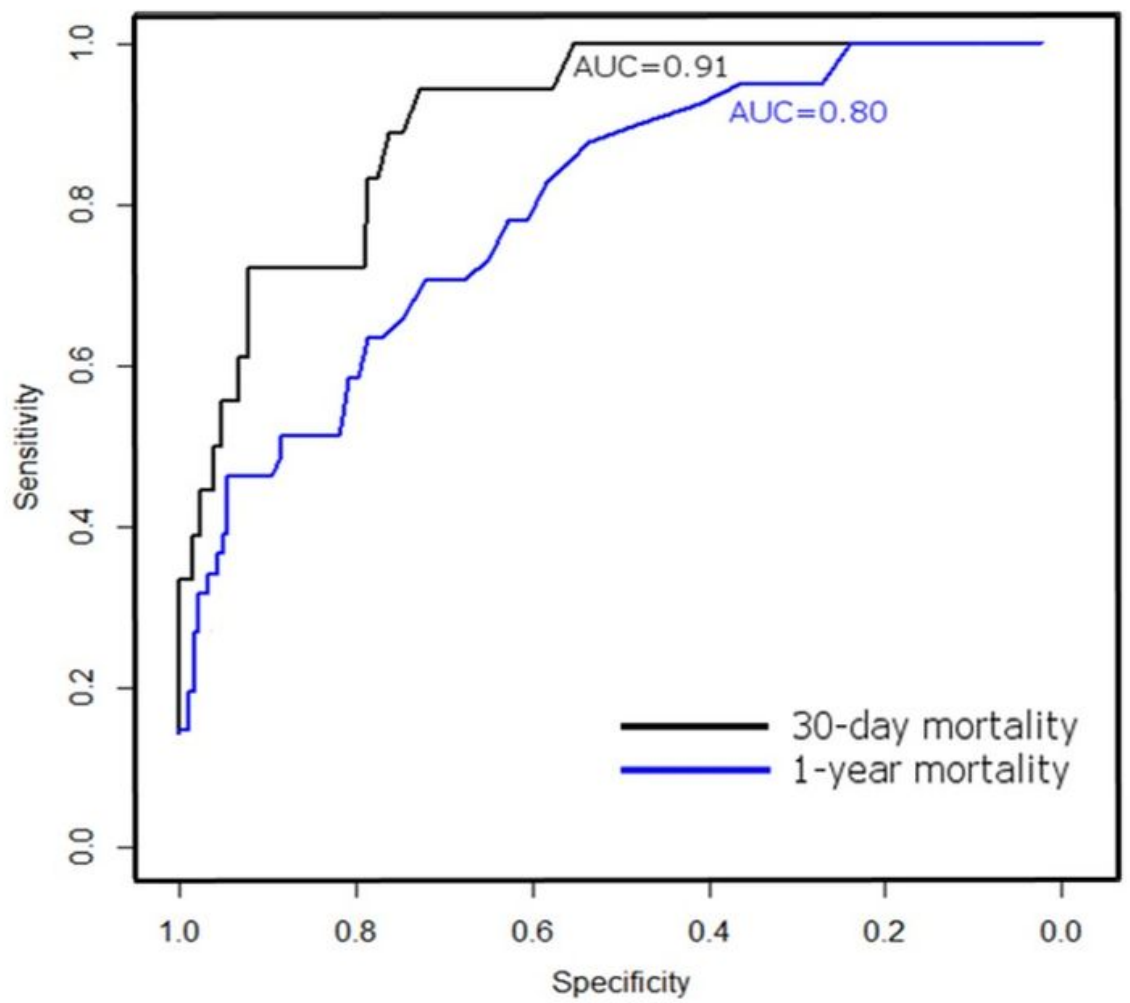

Figure 2 
Receiver operating characteristic curves of 30-day and 1-year mortality

\section{Supplementary Files}

This is a list of supplementary files associated with this preprint. Click to download.

- LactateJoostenappendix.docx 